\title{
Biomarker Imaging Quality of Life Studies Funding Program
}

\author{
National Cancer Institute
}

\section{Source}

National Cancer Institute. Biomarker Imaging Quality of Life Studies Funding Program.

NCI Thesaurus. Code C83476.

A funding mechanism and prioritization process for essential correlative studies and quality of life studies that are incorporated into the fundamental design of a clinical trial. The objective of this initiative is to ensure that the most important biomarker, imaging, and quality of life studies can be initiated in a timely manner in association with late phase clinical trials. 